\title{
Uso de la Inteligencia Artificial para Incrementar la Autonomía de un Robot Móvil Mindstorms NXT en Tareas de Clasificación de Patrones
}

\author{
Abraham Esteban Gamarra Moreno ${ }^{1}$, Juan Gamarra Moreno², Job Daniel Gamarra Moreno³ \\ ${ }^{1}$ Universidad Nacional del Centro del Perú, Av. Mariscal Castilla №3909, Huancayo, Perú \\ 2 Universidad Nacional Mayor de San Marcos, Calle Germán Amézaga № 375, Lima, Perú \\ 3 Universidad Continental, Av. San Carlos 1980, Huancayo, Perú
}

Recibido el 16 de junio del 2018. Aceptado el 5 de julio del 2018

DOI: https://doi.org/10.33017/RevECIPeru2018.0008/

\section{Resumen}

La inteligencia artificial es un área que intenta dotar de inteligencia a las máquinas y entre los tópicos que desarrolla están los sistemas expertos, la lógica difusa, los sistemas de planificación, los algoritmos de búsqueda, la computación evolutiva, redes neuronales artificiales entre otros. Los tópicos de la inteligencia artificial que utiliza este artículo son la visión artificial y las redes neuronales artificiales; además utiliza el microbot o robot móvil Mindstorms NXT, que tiene una capacidad limitada en el procesamiento, así como en el almacenamiento de información. La limitación del robot móvil se da porque no tiene a bordo un computador potente para procesar los algoritmos de visión artificial y de las redes neuronales artificiales; por lo que se utiliza un computador externo para realizar su control a través de la tecnología bluetooth. El procesamiento de los algoritmos de visión artificial y de redes neuronales artificiales se realiza en el computador externo y las acciones que ejecuta el robot móvil son enviadas a este, a través de la comunicación bluetooth. El artículo considera que existe autonomía en un robot móvil, cuando este realiza sus acciones sin intervención humana y los indicadores seleccionados para medir esta autonomía son la localización autónoma de los patrones a reconocer y el reconocimiento autónomo o clasificación de estos patrones. La implementación de la localización autónoma de los patrones a reconocer utiliza sensores ópticos, sensores ultrasónicos y el lenguaje de programación C\#; así como el reconocimiento autónomo de patrones utiliza una cámara inalámbrica ubicada en el robot móvil, algoritmos de visión artificial, redes neuronales artificiales y el lenguaje de programación visual basic .NET. Los resultados muestran que el promedio del indicador porcentaje de patrones localizados en forma correcta en el entorno por el robot móvil Mindstorms NXT es de $37.81 \%$ cuando no se usa la inteligencia artificial y es de $97.18 \%$ cuando se usa la inteligencia artificial. Además, el promedio del indicador porcentaje de patrones reconocidos en forma correcta en el entorno por el robot móvil Mindstorms NXT es de $46.25 \%$ cuando no se usa la inteligencia artificial y es de $96.87 \%$ cuando se usa la inteligencia artificial.

Descriptores: inteligencia artificial, visión artificial, redes neuronales, clasificación de patrones, sensores ópticos, sensores de ultrasonido, microbots, Mindstorms NXT.

\section{Abstract}

Artificial intelligence is an area that tries to equip the machines with intelligence and among the topics developed are expert systems, fuzzy logic, planning systems, search algorithms, evolutionary computation, artificial neural networks among others. The topics of artificial intelligence used in this article are artificial vision and artificial neural networks; also uses the microbot or mobile robot Mindstorms NXT, which has a limited capacity in the processing, as well as in the storage of information. The limitation of the mobile robot is because it does not have a powerful computer on board to process artificial vision algorithms and artificial neural networks; so an external computer is used to perform its control through bluetooth technology. The processing of artificial vision algorithms and artificial neural networks is done on the external computer and the actions performed by the 
mobile robot are sent to it, through bluetooth communication. The article considers that there is autonomy in a mobile robot, when it performs its actions without human intervention and the indicators selected to measure this autonomy are the autonomous localization of the patterns to be recognized and the autonomous recognition or classification of these patterns. The implementation of the autonomous localization of the patterns to be recognized uses optical sensors, ultrasonic sensors and the $C$ \# programming language; as well as the autonomous recognition of patterns uses a wireless camera located in the mobile robot, artificial vision algorithms, artificial neural networks and the visual basic .NET programming language. The results show that the average of the indicator percentage of patterns correctly located in the environment by the Mindstorms NXT mobile robot is $37.81 \%$ when artificial intelligence is not used and it is $97.18 \%$ when artificial intelligence is used. In addition, the average of the indicator percentage of patterns correctly recognized in the environment by the Mindstorms NXT mobile robot is $46.25 \%$ when artificial intelligence is not used and is $96.87 \%$ when using artificial intelligence.

Keywords: artificial intelligence, artificial vision, artificial neural networks, pattern classification, optical sensors, ultrasound sensors, microbots.

\section{Introducción}

En nuestros días la creación de nuevos equipos electrónicos y la disminución de sus costos ha permitido desarrollar la automatización y control de las máquinas, entre ellos se ha desarrollado la robótica como un área que muestra grandes resultados; sobre todo en la robótica industrial, aunque la mayoría de las aplicaciones de hoy utilizan computadoras potentes para poder procesar su información.

Las aplicaciones en la robótica apuntan a utilizar cada vez mejores equipos electrónicos y software especializado; pero existe un área de la robótica que trabaja con robots pequeños denominados robots móviles o microbots que tienen una capacidad limitada tanto en el procesamiento, como en el almacenamiento de información y es ahí a donde se orienta este artículo. Las limitaciones que tienen estos microbots se da porque no tienen a bordo un computador potente para procesar los algoritmos de la inteligencia artificial, específicamente los algoritmos de la visión artificial y de las redes neuronales artificiales, por lo que en este artículo se utiliza un computador externo para realizar el control del robot a través de la tecnología del bluetooth. El procesamiento de los algoritmos de visión artificial y de redes neuronales artificiales se realiza en el computador externo y las acciones que ejecuta el robot móvil son enviadas a este, a través de la comunicación bluetooth.

Con respecto a los antecedentes que tratan la navegación autónoma y el uso de las Redes Neuronales, Pomerleau [1] implementa un sistema de aprendizaje llamado ALVINN (Autonomous Land Vehicle In a Neural Network), donde se presenta una arquitectura de una red neuronal y las técnicas de entrenamiento que permiten a ALVINN conducir en una variedad de circunstancias al vehículo Navlab, incluyendo carreteras pavimentadas y carreteras sin pavimentar; además permite conducir en carreteras con líneas y carretera sin líneas; también permite conducir en carreteras con obstáculos y con obstáculos fuera de la carretera. Este trabajo utiliza una computadora de escritorio llamado Sun Sparcstations para procesar los algoritmos de la Red Neuronal [1]. En otro trabajo de investigación concerniente a la navegación de robots móviles, Stemm [2], implementa un sistema de visión que utiliza redes neuronales artificiales para clasificar objetos y para predecir distancias, complementando con la segmentación de imágenes. Este sistema se implementa en un Robot Móvil Autónomo llamado Xavier, el cual utiliza tres computadoras conectadas en red, donde dos computadoras son Pentium y la otra es una laptop.

Sobre los métodos de aprendizaje para navegación en minirobots móviles, Ramirez [3] propone: "Un algoritmo de aprendizaje para que un minirobot móvil pueda navegar en un ambiente desconocido, justifica su validez y demuestra que se puede asimilar a una red neuronal asociativa; también presenta una metodología formal para entrenar la red neuronal". El minirobot utiliza dos sensores ultrasónicos, un microcontrolador PIC16F84A y adicionalmente tiene una memoria serial tipo EEPROM(Electrically-Erasable Programmable Read-Only Memory) de característica no volátil [3].

Sobre el autoguiado de robots móviles Marcos et al. [4] muestran que: "El objetivo de su trabajo de investigación es la implementación de una estrategia de autoguiado de un robot móvil en entornos desconocidos, empleando como núcleo de decisión una red neuronal". Además Marcos et al. [4]: "Desarrollan un entorno en Matlab para la generación de trayectorias de entrenamiento, el 
entrenamiento propiamente dicho y la simulación de la red. En la fase de desarrollo se ha tenido en cuenta las peculiaridades del microbot PICBOT3, microbot sobre el cual se implementa la red neuronal". EI PICBOT3 es un microbot que utiliza el microcontrolador PIC16F876; este microcontrolador tiene una capacidad de hasta $4 \mathrm{~K}$ en programas y dispone de 33 líneas de E/S de propósito general. Para el trabajo de investigación los autores utilizaron cinco sensores de ultrasonidos para detectar la presencia de obstáculos, los cuales estaban distribuidos en la parte delantera [4].

Con respecto a los Sistemas de Visión Artificial, Sobrado [5] en su trabajo de investigación implementa: "Un sistema robótico automatizado para el reconocimiento y localización de piezas. El objetivo de su trabajo es implementar un sistema que permita identificar piezas depositadas en una mesa, para luego encontrar su localización exacta y realizar una tarea de pick \& place mediante un manipulador de cinco grados de libertad, llamado SCORBOTER-IX.". EI reconocimiento se realiza mediante una Red Neuronal del tipo Backpropagation.

Con respecto al robot Mindstorm NXT en Robotica.com [6], se muestra la construcción de 4 modelos del Robot Mindstorms NXT 2.0: el primer modelo es un vehículo que cuida una habitación; el segundo modelo es un robot animal, con forma de cocodrilo que salta y muerde si alguien se acerca; el tercer modelo es un robot que elige objetos por el color; el cuarto modelo es un robot humanoide. Los robots construidos, reaccionan de acuerdo a la percepción que realizan sus sensores y no utilizan ningún tópico de la Inteligencia Artificial para decidir la acción a realizar.

Según mindsensors.com [7] existe: "El Sistema de visión v3 para NXT (NXTCam-v3), para aplicaciones de visión artificial con Mindstorms NXT. Este subsistema de Visión tiene la capacidad de procesamiento de imágenes en tiempo real para detectar y rastrear una línea o diferenciar objetos de hasta 8 colores, informa de las coordenadas de cada objeto a NXT". "El uso de NXTCam requiere conocimientos técnicos de programación y capacidad analítica, y es ideal para eventos como la Robocup Junior, competiciones de robots, proyectos de final de carrera, investigación, etc" [7]. La cámara se incorpora al Robot Mindstorms NXT a través de un puerto asignado a los sensores; pero las limitaciones de este subsistema es que solamente reconoce líneas o diferencia colores, pero no realiza otros procesos de visión artificial.
Otro trabajo de investigación que utiliza la cámara NXTCam incorporada al Robot Mindstorms NXT, es presentado por Novischi [8]; donde presenta dos robots Mindstorms NXT que tienen la capacidad de evitar obstáculos desde un punto inicial a un punto final. El primer robot usa un sensor ultrasónico y el segundo robot usa el subsistema de visión incorporada en la cámara. Este trabajo de investigación igual que el anterior utiliza la cámara para realizar el reconocimiento de objetos a través del color. Otro trabajo de investigación con Mindstorms NXT que utiliza una cámara para procesos básicos de Visión Artificial es el trabajo de Hartog [9], donde se integra una cámara CMUCam3 al Robot Mindstorms que permite reconocer esferas de colores específicos. Para realizar la comunicación con el Robot de la cámara CMUCam3 se necesita conectar una tarjeta electrónica adicional. La aplicación sólo realiza reconocimiento de objetos en función al color del objeto.

Sobre la creación de un robot móvil explorador con LEGO® Mindstorms ${ }^{\circledR}$ NXT, la página web nxtprograms.com [10] implementa un robot cuya misión es explorar alrededor de un cuarto, casa o donde desee explorar. El robot intenta "ver" a través de sensores ultrasónicos las paredes y otros obstáculos y evita ir hacia ellos; entonces gira su cabeza a la derecha e izquierda para "mirar" en qué dirección debe desplazarse. En el caso que se desplace accidentalmente hacia un objeto, se tiene un bumper en el frente, para hacer que el robot se detenga y decida adonde debe girar. La construcción de esta aplicación no utiliza la Inteligencia Artificial para evitar los obstáculos [10].

Sobre el uso de la visión por computador, utilizando el software Roborealm [11], aplicado a robots móviles, en el tutorial Adding vision to the Lego, en el sitio de Roborealm se muestra como modificar el robot Lego Mindstorms NXT, para que pueda usar la Visión por computador; con el objetivo de encontrar una bola azul, coger este y moverlo hacia un cono naranja. Los objetos son reconocidos utilizando un filtro de color. Otra implementación del uso de la visión por computador, utilizando el software Roborealm aplicado a los robots móviles, se encuentra en el tutorial Ilamado Lego PC Bot, donde se muestra la implementación de un robot PC, construido con un kit Lego Mindstorms NXT; con el objetivo de crear un sistema de procesamiento de visión a bordo, que consta de una mini laptop y una cámara web dispuesto en la parte superior del robot. Para mover el robot alrededor del cuarto y a la vez evitar los obstáculos se utiliza el 
color del piso y se evitan los obstáculos que no son del color del piso [11].

Con respecto al uso de la Inteligencia Artificial en robots Mindstorms NXT, Klassner y McNally [12], muestran las posibilidades de utilizar la plataforma NXT en el currículo de Ciencias de la Computación e Inteligencia artificial; específicamente en los temas de odometría, planificación de caminos, localización y construcción de mapas. La plataforma NXT es el cerebro del robot que incluye un sistema de archivos local sobre $256 \mathrm{~Kb}$ de memoria flash RAM, 64 Kb RAM para ejecución de programas, un CPU ARM7 de $48 \mathrm{MHz}$ con soporte para comunicación con Bluetooth. Además, incluye una pantalla LCD de $100 \times 64$ pixeles, puerto USB 2.0 para cargar programas desde una PC, 4 puertos para sensores y tres puertos para motores.

Observando las técnicas utilizadas en los trabajos previos se puede observar que los robots móviles utilizan computadoras de escritorio o laptops a bordo para realizar las tareas de navegación y de reconocimiento de objetos; inclusive existen dos robots conocidos que se han difundido en estos últimos años, uno de ellos es el Robot mascota Aibo de Sony, que tiene forma de perro y que dispone de sensores, cámaras e Inteligencia Artificial; pero tiene una computadora a bordo. El otro robot es ASIMO (acrónimo de "Advanced Step in Innovative Mobility"- paso avanzado en movilidad innovadora) y es un robot humanoide creado por la empresa Honda; pero también su característica principal es su elevado costo y tiene un computador a bordo.

Este artículo no utiliza un robot móvil con un computador de escritorio o laptop a bordo de este, en su lugar utiliza el robot Mindstorms NXT que tiene capacidad limitada en el procesamiento, así como en el almacenamiento de información; además tiene un peso aproximado de $500 \mathrm{gr}$ y un costo bajo en comparación con los robots mencionados en el párrafo anterior. Aun teniendo en cuenta las limitaciones del robot Mindstorms NXT, se ha incorporado a este, desde el exterior, dos tópicos de inteligencia artificial, específicamente la visión artificial y las redes neuronales artificiales; con el objetivo de mejorar la autonomía del robot en tareas de clasificación de patrones.

El artículo muestra que la inclusión al robot Mindstorms NXT de los dos tópicos inteligencia artificial, específicamente la visión artificial y redes neuronales artificiales, permite realizar localizar el patrón a reconocer y reconocer o clasificar ese patrón sin intervención humana.

\section{Metodología}

Este artículo considera que la autonomía del robot Mindstorms NXT está en función del uso de la Inteligencia Artificial:

\section{Autonomía del robot $=f($ Inteligencia Artificial $)$}

También el artículo define que la inteligencia artificial es la emulación de las facultades mentales de las personas, para incorporarlos a las máquinas. Los tópicos que desarrolla la inteligencia artificial son: los sistemas expertos, la lógica difusa, los sistemas de planificación, los algoritmos de búsqueda, la computación evolutiva, redes neuronales artificiales entre otros. Los tópicos elegidos en este artículo son la visión artificial y las redes neuronales artificiales.

Luego de la elección de los tópicos seleccionados la expresión de la autonomía queda de la siguiente forma:

Autonomía del robot $=f$ (Visión artificial, Redes Neuronales Artificiales)

La autonomía del robot Mindstorms NXT se define como la capacidad del robot de interactuar con su entorno y de adaptarse a los posibles cambios sin la necesidad de un operador humano. En este caso la autonomía del robot se da cuando localiza el patrón a reconocer y cuando reconoce o clasifica ese patrón. La autonomía del robot Mindstorms NXT se mide a través de los siguientes indicadores:

Porcentaje de
patrones localizados
en forma correcta en el
entorno del robot móvil
Mindstorms NXT
Porcentaje de Rutonomía del
patrones reconocidos
en forma correcta en el
entorno por el robot
móvil Mindstorms NXT

Para determinar si un patrón es localizado en forma correcta en el entorno del robot móvil Mindstorms Nxt, el robot móvil se desplaza a través de la línea negra que se muestra en la figura 1 y cada vez que localice el cartel que contiene al patrón a reconocer se detiene. Si se detiene al frente del cartel del 
patrón a reconocer y el patrón queda al centro del enfoque de la cámara se considera que se ha localizado el patrón en forma correcta.

Para determinar si un patrón es reconocido en forma correcta en el entorno por el robot móvil Mindstorms NXT, se obtiene la imagen que tiene enfocado la cámara y si el sistema de visión artificial con red neuronal lo clasifica como uno de los patrones válidos, se considera un reconocimiento correcto.

Debido a que el robot Mindstorms NXT tiene una capacidad limitada de memoria y una capacidad limitada de ejecución de programas, se vuelven intratables procesar los algoritmos de la inteligencia artificial en el robot; específicamente los algoritmos de visión artificial y de redes neuronales artificiales. Para salvar esta situación se propone interconectar al robot Mindstorms NXT a través del dispositivo bluetooth con una laptop externa; además, se instala una cámara inalámbrica en el robot y el digitalizador de la cámara inalámbrica se instala en la laptop tal como se muestra en la figura 1.

La implementación del sistema de localización autónoma de los patrones a reconocer utiliza un sensor óptico, un sensor ultrasónico y el lenguaje de programación C\#. Para lograr la localización autónoma de los patrones se ha desarrollado un programa que permite ordenar al robot a seguir la línea negra, identificar la posición del cartel y detener el robot frente a este cartel, de tal forma que el patrón quede enfocado en el centro de la cámara. La lectura de los sensores se envía desde el robot hacia la laptop a través de la comunicación bluetooth, la laptop procesa el valor de los sensores y envía órdenes a través de la comunicación bluetooth a los motores del robot para avanzar, girar o detenerse según sea el caso.

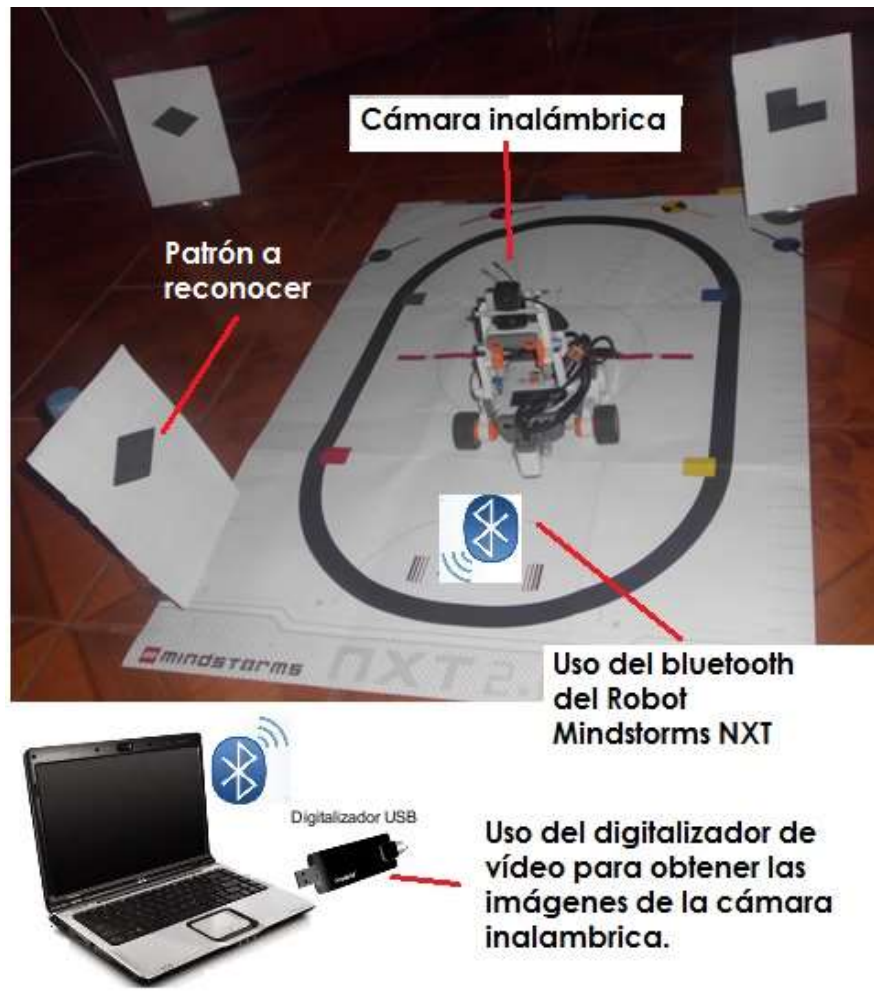

Figura 1: Esquema de comunicación entre el robot Mindstorms NXT y la laptop externa al robot.

El reconocimiento de los patrones se realiza con un programa en la laptop externa al robot, que capta la imagen de la cámara inalámbrica a través del digitalizador; procesa la imagen utilizando las técnicas de visión artificial y redes neuronales artificiales e identifica algún patrón valido.

El diagrama de flujo de la localización de los patrones a reconocer y del reconocimiento 0 clasificación de los patrones se muestra en la figura 2.

El proceso de la visión artificial en este artículo se realiza siguiendo las etapas de la figura 3 [13]. El proceso de la visión artificial inicia con la adquisición de la imagen través de la cámara inalámbrica, continua con el preprocesamiento donde realiza el suavizado de la imagen, conversión de la imagen a blanco y negro y reducción de la imagen; luego en la segmentación y descripción se realiza el reconocimiento de los patrones utilizando redes neuronales artificiales. 


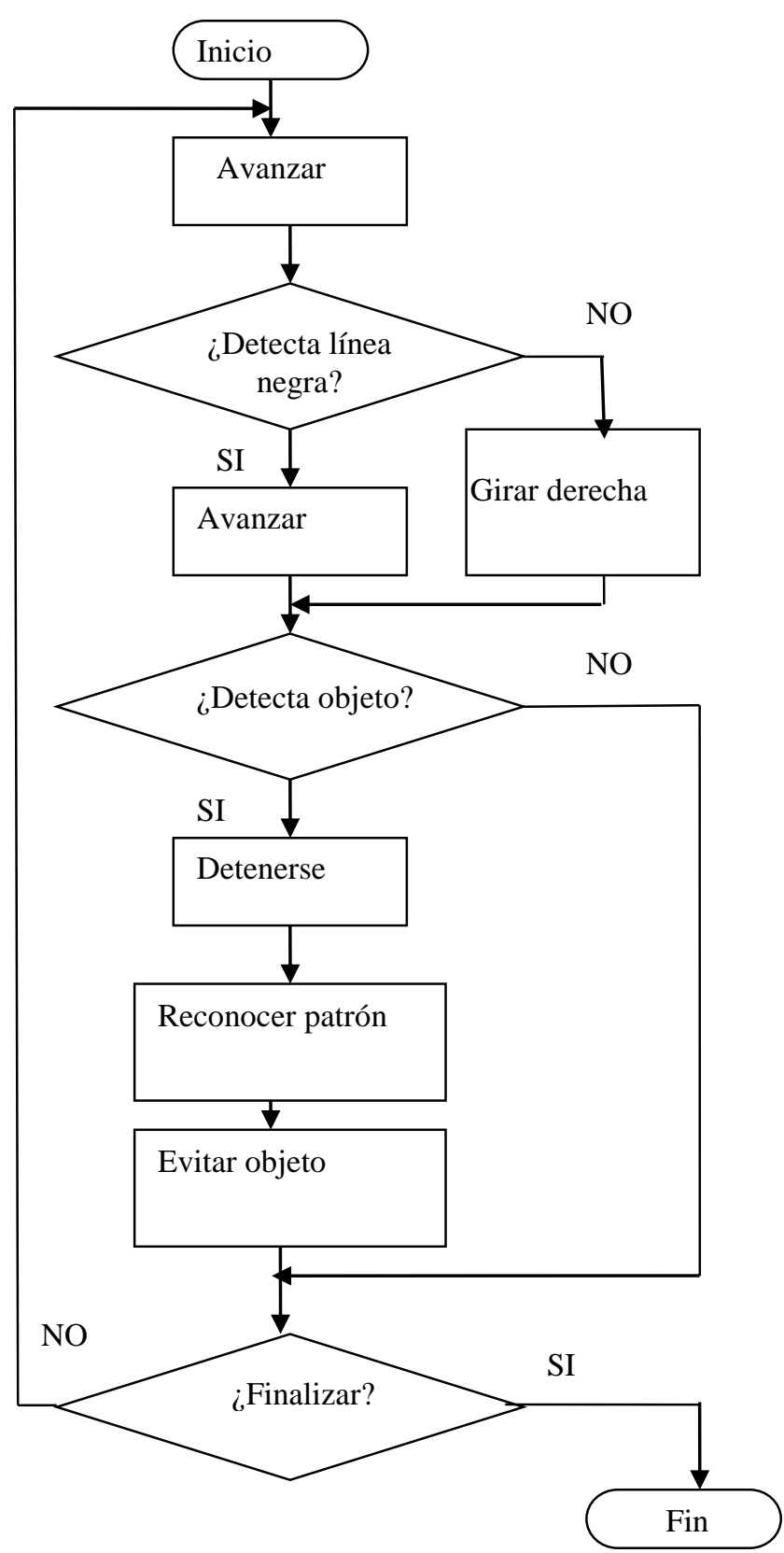

Figura 2: Diagrama de flujo de la localización de los patrones a reconocer $y$ del reconocimiento 0 clasificación de los patrones.

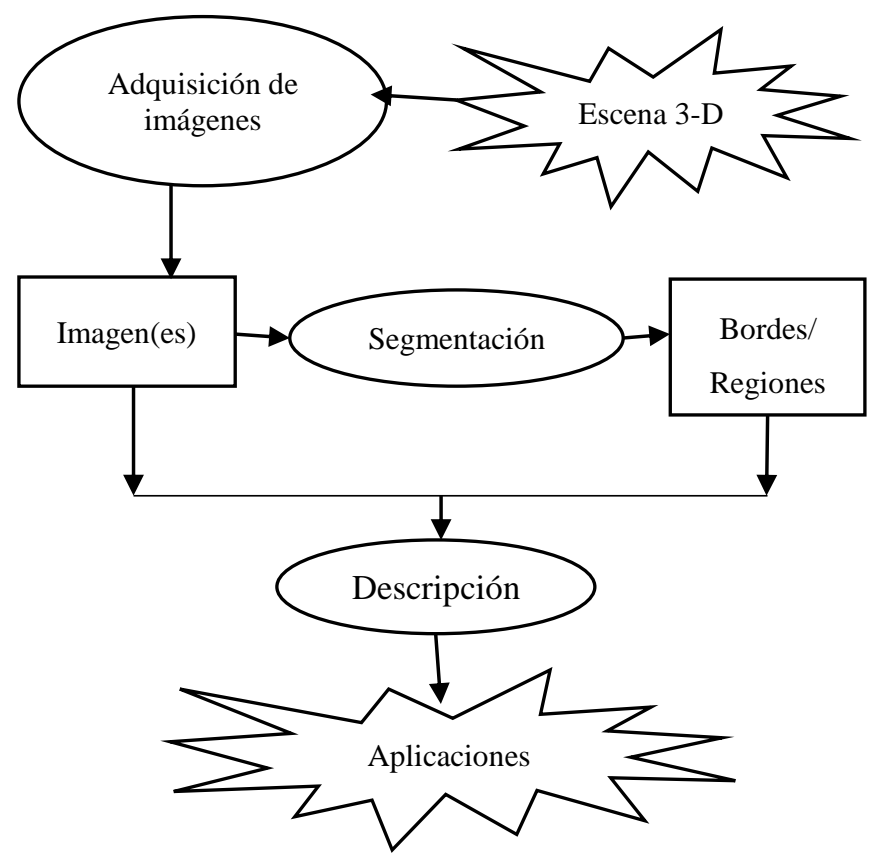

Figura 3: Etapas de la visión artificial.

La preparación de los datos para entrenar la red neuronal inicia con 10 imágenes de cada patrón, con una resolución de la imagen de 720 x 480 pixeles, se suaviza estos patrones; una vez realizado el suavizado son transformados a blanco y negro. Una vez que se tiene los patrones en blanco y negro, ahora se procede a reducirlos a una resolución de $18 \times 12$ pixeles. Luego de obtener la reducción se convierten las imágenes a ceros y unos de tal forma que se preparen como patrones de entrenamiento de la red neuronal.

En este artículo se ha elaborado un programa en Visual Basic .NET que toma las imágenes reducidas de 18 x 12 pixeles y lleva estos pixeles de una representación matricial a una representación de una sola dimensión de 216 ceros o unos y añade una columna que almacena el valor de 1 o 2 que identifica al patrón a identificar; luego los guarda en un archivo de texto.

La estructura que deben tener los patrones para el entrenamiento de la red neuronal se muestra en la tabla 1. Cada fila tiene la representación de una imagen en ceros y unos, según corresponda. El valor de 0 representa a un pixel negro y el valor de 1 representa a un pixel blanco.

La red neuronal utilizada tiene 216 neuronas en la capa de entrada, 113 neuronas en la capa oculta y 1 neurona en la capa de salida, tal como se muestra en la figura 4. La cantidad de neuronas en la capa oculta se calcula a través de la siguiente expresión: 


$$
\begin{gathered}
\text { \# neuronas ocultas }=\frac{\text { entradas }+ \text { salidas }}{2}+\sqrt{\# \text { patrones }} \\
\text { \# neuronas ocultas }=\frac{216+1}{2}+\sqrt{20}=113
\end{gathered}
$$

Tabla 1: Patrones de entrenamiento que utiliza la red neuronal.

\begin{tabular}{|c|c|c|c|c|l|}
\hline $\mathrm{X} 1$ & $\mathrm{X} 2$ & $\ldots$ & $\mathrm{X} 216$ & salida & encabezado \\
\hline 1 & 1 & $\ldots$ & 1 & 1 & $\begin{array}{l}\text { imagen } \\
\text { reducida 1 }\end{array}$ \\
\hline 1 & 1 & $\ldots$ & 1 & 1 & $\begin{array}{l}\text { imagen } \\
\text { reducida 2 }\end{array}$ \\
\hline 1 & 1 & $\ldots$ & 0 & 1 & $\begin{array}{l}\text { imagen } \\
\text { reducida 3 }\end{array}$ \\
\hline$\ldots$ & $\ldots$ & $\ldots$ & $\ldots$ & $\ldots$ & $\ldots$ \\
\hline 1 & 1 & $\ldots$ & 1 & 2 & $\begin{array}{l}\text { imagen } \\
\text { reducida 19 }\end{array}$ \\
\hline 1 & 1 & $\ldots$ & 0 & 2 & $\begin{array}{l}\text { imagen } \\
\text { reducida 20 }\end{array}$ \\
\hline
\end{tabular}

El entrenamiento de la red neuronal se realizó con el software Neuroshell, este software permite tomar los patrones de entrenamiento, luego calcula los pesos adecuados para la red neuronal y finalmente genera un procedimiento en el código de visual basic. NET, el cual representa a la red neuronal entrenada.

El uso de la red neuronal artificial entrenada, que se encuentra en el procedimiento de visual basic, requiere que se envíe al procedimiento un patrón de entrada con 256 valores de unos o ceros que representa a la imagen y recibe como respuesta un valor cercano a uno o dos que representa el valor del patrón a clasificar

Para utilizar el procedimiento en visual basic, generado por el software Neuroshell, se ha implementado en el sistema de visión artificial un módulo que obtiene la imagen de la cámara inalámbrica que se encuentra en el robot con una resolución de 720 × 480 pixeles, lo suaviza, lo convierte a blanco y negro, reduce la resolución a $18 \times 12$ pixeles y luego lo convierte a unos y ceros. Una vez que se tenga la imagen de la cámara en unos y ceros, se pasa estos valores al procedimiento, el cual clasifica si pertenece al patrón 1 o al patrón 2 .

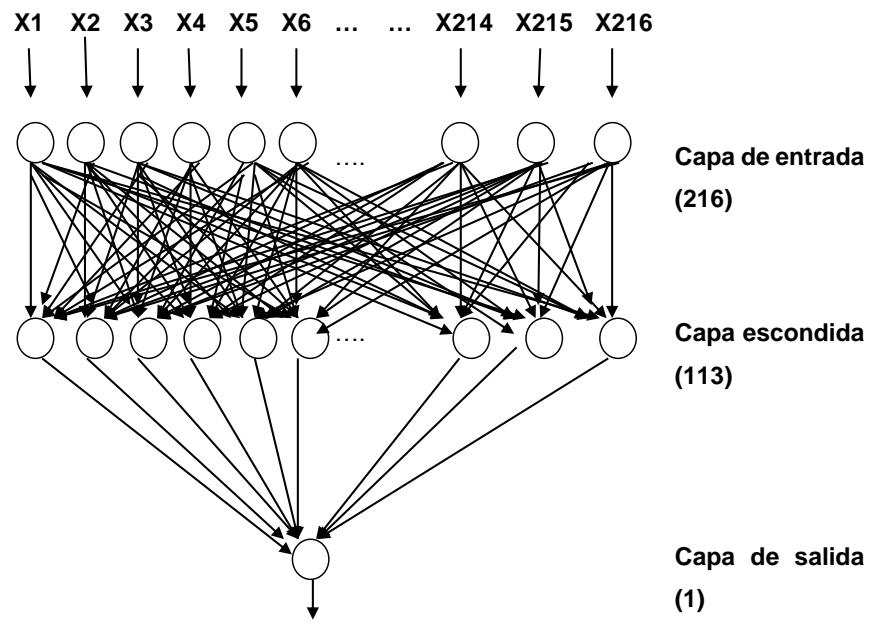

Figura 4: Estructura de la red neuronal utilizada para el reconocimiento de los patrones.

Cabe resaltar que la red neuronal artificial solamente clasifica si la imagen pertenece al patrón 10 al patrón 2.

\section{Resultados}

En este artículo se utiliza la visión artificial y una red neuronal artificial; y como estos son tópicos de la inteligencia artificial, se considera que la investigación ha utilizado la inteligencia artificial para incrementar la autonomía de un robot móvil Mindstorms NXT en tareas de clasificación de patrones.

La tabla 2 muestra el porcentaje promedio de los indicadores de la variable autonomía de un robot móvil Mindstorms NXT (patrones localizados en forma correcta y patrones reconocidos en forma correcta), con el uso de la Inteligencia Artificial y sin el uso de la Inteligencia Artificial. Además, en la figura 5 se muestra el gráfico de barras que muestra el porcentaje promedio de los indicadores de la variable autonomía de un robot móvil Mindstorm NXT (patrones localizados en forma correcta y patrones reconocidos en forma correcta), con el uso de la Inteligencia Artificial y $\sin$ el uso de la Inteligencia Artificial. 
Al observar la tabla 2, se desprende que el promedio del indicador porcentaje de patrones localizados en forma correcta en el entorno por el robot móvil Mindstorms NXT es de $37.81 \%$ cuando no se usa la inteligencia artificial y es de $97.18 \%$ cuando se usa la inteligencia artificial. Además, el promedio del indicador porcentaje de patrones reconocidos en forma correcta en el entorno por el robot móvil Mindstorms NXT es de $46.25 \%$ cuando no se usa la inteligencia artificial y es de $96.87 \%$ cuando se usa la inteligencia artificial.

La figura 5 muestra el gráfico de barras donde se puede observar que el promedio del indicador porcentaje de patrones localizados en forma correcta en el entorno por el robot móvil Mindstorms NXT es menor cuando no se usa la inteligencia artificial que cuando se usa la inteligencia artificial. También se tiene que el promedio del indicador porcentaje de patrones reconocidos en forma correcta en el entorno por el robot móvil Mindstorms NXT es menor cuando no se usa la inteligencia artificial que cuando se usa la inteligencia artificial.

Además, se tiene en cuenta que los indicadores porcentaje de objetos localizados en forma correcta en el entorno por el robot móvil Mindstorms NXT y porcentaje de objetos reconocidos en forma correcta en el entorno por el robot móvil Mindstorms NXT, en conjunto miden la variable autonomía de un robot móvil Mindstorms NXT y que al aumentar los valores de estos indicadores también aumentan la autonomía del robot.

\section{Interpretación de los resultados}

La mayoría de las aplicaciones para el control de los robots móviles que hacen uso de la inteligencia artificial, específicamente la visión artificial y las redes neuronales artificiales, tienen una computadora de escritorio a bordo, el cual toma la decisión de la acción a ejecutar [1]; pero el presente trabajo de investigación no utiliza ningún computador de escritorio a bordo.

Tabla 2: Porcentaje promedio de los indicadores de la variable autonomía de un robot móvil Mindstorm Nxt (patrones localizados en forma correcta $y$ patrones reconocidos en forma correcta), con el uso de la Inteligencia Artificial y sin en el uso de la Inteligencia Artificial.

\begin{tabular}{|c|c|c|c|}
\hline \multirow{7}{*}{\multicolumn{2}{|c|}{$\begin{array}{l}\text { Uso de la inteligencia } \\
\text { artificial (visión artificial y } \\
\text { redes neuronales) } \\
\text { (1= no se usa IA, } 2=\text { se usa } \\
\text { IA) }\end{array}$}} & \multicolumn{2}{|c|}{$\begin{array}{c}\text { Autonomía de un robot móvil } \\
\text { Mindstorms NXT }\end{array}$} \\
\hline & & \multirow{6}{*}{$\begin{array}{l}\text { Porcentaje de } \\
\text { patrones } \\
\text { localizados en } \\
\text { forma correcta } \\
\text { en el entorno } \\
\text { por el robot } \\
\text { móvil } \\
\text { Mindstorms } \\
\text { NXT }\end{array}$} & \multirow{6}{*}{$\begin{array}{l}\text { Porcentaje de } \\
\text { patrones } \\
\text { reconocidos } \\
\text { en forma } \\
\text { correcta en el } \\
\text { entorno por el } \\
\text { robot móvil } \\
\text { Mindstorms } \\
\text { NXT }\end{array}$} \\
\hline & & & \\
\hline & & & \\
\hline & & & \\
\hline & & & \\
\hline & & & \\
\hline \multirow[t]{3}{*}{1} & Media & 37,8125 & 46,2500 \\
\hline & $\mathrm{N}$ & 40 & 40 \\
\hline & Desv. típ. & 16,62354 & 22,32482 \\
\hline \multirow[t]{3}{*}{2} & Media & 97,1875 & 96,8750 \\
\hline & $\mathrm{N}$ & 40 & 40 \\
\hline & Desv. típ. & 5,99646 & 8,37330 \\
\hline \multirow[t]{3}{*}{ Total } & Media & 67,5000 & 71,5625 \\
\hline & $\mathrm{N}$ & 80 & 80 \\
\hline & Desv. típ. & 32,35239 & 30,48753 \\
\hline
\end{tabular}

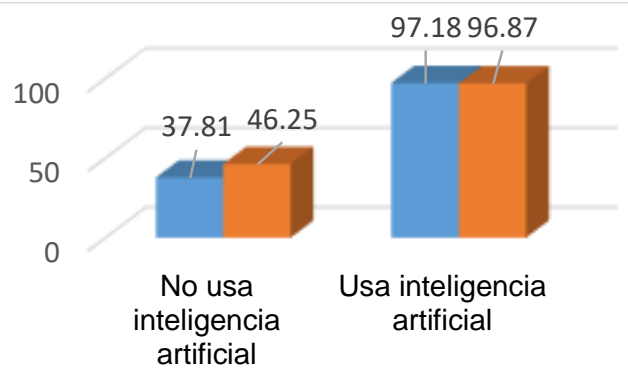

- Porcentaje de patrones localizados en forma correcta en el entorno por el robot móvil Mindstorms Nxt

Figura 5: Gráfico de barras que muestra el porcentaje promedio de los indicadores de la variable autonomía de un robot móvil Mindstorm Nxt (patrones localizados en forma correcta y patrones reconocidos en forma correcta), con el uso de la Inteligencia Artificial y sin el uso de la Inteligencia Artificial.

De otra parte se tiene que la implementación de la navegación de minirobots o microbots, utilizan redes neuronales y microcontroladores PIC16F84A, 
PIC16F876 y sensores de ultrasonido; pero esos trabajos no utilizan la visión artificial [3, 4].

También se tienen algunos trabajos de investigación que incluyen un procesamiento básico de visión artificial en robots móviles 0 microbots, esto se realiza con el uso de cámaras que tienen empotrados los algoritmos básicos de visión artificial; pero el reconocimiento de objetos solamente está basado en el color del objeto [6 -11].

En este artículo se utiliza robots móviles o microbots que tienen una capacidad limitada tanto en el procesamiento, como en el almacenamiento de información. La limitación del robot móvil se da porque no tienen a bordo un computador potente para procesar los algoritmos de visión artificial y de las redes neuronales artificiales; por lo que se ha utilizado un computador externo para realizar su control a través de la tecnología bluetooth. El procesamiento de los algoritmos de visión artificial y de redes neuronales artificiales se realiza en el computador externo y las acciones que ejecuta el robot móvil son enviadas a este, a través de la comunicación bluetooth.

\section{Conclusiones}

Para mejorar la autonomía Robot móvil Mindstorm Nxt en tareas de clasificación de objetos se utilizó sensores ópticos, sensores ultrasónicos y se implementó un sistema de visión artificial con redes neuronales en Visual Basic .NET.

El sistema de visión artificial utiliza una cámara inalámbrica ubicada en el robot y la recepción se realiza en una laptop externa al robot.

El procesamiento del sistema de visión artificial con redes neuronales artificiales se realiza en forma externa, en una laptop y además se controla al robot Mindstorms NXT a través de la comunicación bluetooth utilizando el lenguaje $\mathrm{C \#}$.

El reconocimiento de los patrones se realiza a través del uso de redes neuronales artificiales implementado en el lenguaje Visual Basic .NET.

Los resultados muestran que el promedio del indicador porcentaje de patrones localizados en forma correcta en el entorno por el robot móvil Mindstorms NXT es de $37.81 \%$ cuando no se usa la inteligencia artificial y es de $97.18 \%$ cuando se usa la inteligencia artificial. Además, el promedio del indicador porcentaje de patrones reconocidos en forma correcta en el entorno por el robot móvil Mindstorms NXT es de $46.25 \%$ cuando no se usa la inteligencia artificial y es de $96.87 \%$ cuando se usa la inteligencia artificial.

\section{Agradecimientos}

Agradecemos a la facultad de Ingeniería de Sistemas de la Universidad Nacional del Centro del Perú por brindar sus instalaciones en el desarrollo de la investigación.

\section{Referencias}

[1] D.A. Pomerleau, The Springer International Series in Engineering and Computer Science 233 (1993) 19-43.

[2] M. Stemm, B.Sc. Thesis, Carnegie Mellon University, 1994.

[3] G. Ramírez, Dyna 138 (2003) 59-66.

[4] C. Marcos, R. Guzmán and R. Alaiz, XXV Jornadas de Automática, 2004, edited by Ciudad Real (J.A. Somolinos, 2004), p.1.

[5] E. Sobrado, M. Sc. thesis, Pontificia Universidad Católica del Perú, 2003.

[6] Ro-botica, LEGO Mindstorms NXT 2.0., Recuperado de http://www.robotica.com/mindstorms.asp (2011).

[7] Mindsensors, Vision Subsystem v3 for NXT: NXTCam-v3, Recuperado de http://www.mindsensors.com/index.php (2011).

[8] D. Novischi, C. llas, Robotic \& Management 14 (2009) 2.

[9] L. Hartog, Semester thesis, Institut für Technische Informatik und Kommunikationsnetze, 2008.

[10] Nxtprograms, Fun Projects for your $\angle E G O \Theta$ MINDSTORMS ${ }^{A}$ NXT, Recuperado de http://www.nxtprograms.com/index2.html (2011).

[11] Roborealm Tutorials, Recuperado de http://www.roborealm.com/tutorials.php (2011).

[12] F. Klassner and M. McNally, 2007 AAAI Spring Symposium 1 (2007) 103-104.

[13] G. Pajares and J. De la Cruz, Visión por Computador: Imágenes digitales $y$ aplicaciones (Alfa y Omega, México, 2008), p 40.

E-mail: aegamarram@gmail.com 